\title{
Validating Technological Innovation
}



David Coniam • Peter Falvey

\section{Validating Technological Innovation}

The Introduction and Implementation of Onscreen Marking in Hong Kong

自 Springer 


\section{David Coniam}

Department of Curriculum and Instruction

The Education University of Hong Kong

Tai Po, Hong Kong

\author{
Peter Falvey \\ Department of Curriculum and Instruction \\ The Education University of Hong Kong \\ Tai Po, Hong Kong
}

ISBN 978-981-10-0432-2 ISBN 978-981-10-0434-6 (eBook)

DOI 10.1007/978-981-10-0434-6

Library of Congress Control Number: 2016936563

(C) Springer Science+Business Media Singapore 2016

This work is subject to copyright. All rights are reserved by the Publisher, whether the whole or part of the material is concerned, specifically the rights of translation, reprinting, reuse of illustrations, recitation, broadcasting, reproduction on microfilms or in any other physical way, and transmission or information storage and retrieval, electronic adaptation, computer software, or by similar or dissimilar methodology now known or hereafter developed.

The use of general descriptive names, registered names, trademarks, service marks, etc. in this publication does not imply, even in the absence of a specific statement, that such names are exempt from the relevant protective laws and regulations and therefore free for general use.

The publisher, the authors and the editors are safe to assume that the advice and information in this book are believed to be true and accurate at the date of publication. Neither the publisher nor the authors or the editors give a warranty, express or implied, with respect to the material contained herein or for any errors or omissions that may have been made.

Printed on acid-free paper

This Springer imprint is published by Springer Nature

The registered company is Springer Science+Business Media Singapore Pte Ltd. 


\section{Foreword}

Validating Technological Innovation: The Introduction and Implementation of Onscreen Marking in Hong Kong is not only a coherent account of a series of validation studies linked to the implementation of onscreen marking (OSM) in Hong Kong, it is a book representing a 35-year research collaboration of the two authors who have in-depth knowledge about the context of the research. Mostly importantly, it is a book about the future of the field. It is challenging to predict the future in the current world of ours with many uncertainties, but I have no doubt that validation research of onscreen marking will increase, and this book will be read by many researchers for years to come.

As the new millennium was getting underway, in January 2000, Lyle Bachman's article, entitled 'Modern language testing at the turn of the century: assuring that what we count counts', appeared in Language Testing (Volume 17). The article is a 40-plus-page comprehensive review of language testing in the 1980s and 1990s, drawing on more than 200 published works. The personal, retrospective paper focuses on a wide range of areas that developed mainly in the 1990s, including: '(a) research methodology; (b) practical advances; (c) factors that affect performance on language tests; (d) authentic, or performance, assessments; and (e) concerns with the ethics of language testing and professionalising the field' (Bachman 2000, p.4).

Under (b), Bachman lists computer-based assessment as one of practical advances in the field of language testing pointing to the increasing use of computer technology in the development and delivery of language tests. He stated that 'On the practical side, advances in the technology of test design and development, along with the availability and use of ever more sophisticated computer- and web-based applications for test administration, scoring and analysis, have resulted in a greater range of test formats and assessment procedures than has ever been available'. In this, Bachman cautions the field that 'the challenge in applying such technologies to language assessment will be to recognise not only the potential benefits but also the limitations of these technologies, and not let the psychometric and technological tails wag the applied linguistics dog' (p. 9). In discussing the future and what lies ahead, Bachman states that 'the abstract technical debate about dimensionality and the appropriateness of different IRT models has, I believe, been replaced with a 
much more pragmatic focus on practical applications, particularly with respect to performance assessments that involve raters and computer-based tests' (p. 22). Bachman did not specifically discuss onscreen marking, but the above statements clearly direct us to the challenges we are facing in the use of computer-assisted assessment over traditional paper methods. In this sense, this book presenting a coherent series of research studies into onscreen assessment of continuous writing and its impact upon markers' judgements is, therefore, both timely and important.

As the two authors point out, onscreen marking (the marking of examination scripts on screen instead of on paper) is being used much more frequently as technology develops. Over the past 10 years, there has been a worldwide move towards OSM. Indeed, OSM can offer many benefits over traditional paper methods. However, in transferring from one medium to another, it is crucial to understand the extent to which the new medium may alter the nature of traditional assessment practice and affect marking reliability through empirical evidence, evidence that is provided by this book.

This book is unique in a number of ways. First, it charts the development of the validity of OSM in the implementation of OSM across the board in Hong Kong, i.e. in every subject in the public school leaving examination system. The book further provides a series of validation studies over time, which began, in 2009, with the publication of Coniam (2009a), the first description of what became, by 2014/2015, a series of validation studies of OSM in the school public examinations system in Hong Kong, operated by the Hong Kong Examinations and Assessment Authority (HKEAA). In total, nine validation studies were carried out and published by David Coniam, sometimes with co-writers (Alison Sau-chu Yeung [Chap. 7], Zi Yan [Chaps. 10, 12 and 13] and Peter Falvey, the co-author of this book).

Second, the book provides a coherent picture, from a disparate series of validation research studies, backed by useful background information on Hong Kong's education and assessment history, the HKEAA and its work (Chap. 2), a pictorial representation of the OSM process taken from screenshots (Chap. 3) and an introduction to the methodological approaches and tools used in the quantitative and qualitative studies on the OSM process. Therefore, this mixture of background information and research input makes this book extremely useful for readers and other academics within other similar educational contexts. In this sense, the book also provides a useful template for authorities/jurisdictions who want to carry out thorough validation studies in preparation for the wholesale implementation of OSM.

Third, the book is a labour of love from two authors who have had a long, productive association as writers, researchers, teachers, publishers and colleagues stretching over 35 years within the Hong Kong language education context and internationally. Much of this long association started in Hong Kong where they both have an in-depth knowledge of the education and examination system and have established good working relations with the HKEAA and the Education Bureau of the Government of the Hong Kong Special Administrative Region. Such a collaborative connection between test users and test developers and educators and ministries of education is rare in other education contexts. Further, much of the long 
association between the authors has a background in assessment (many books, chapters in books and article publications). David and Peter have spent 20 years on language benchmarking (with a lot of publications) and now OSM. They both have a background with examination authorities (David with the HKEAA where he was an HKEAA officer and long-time examination developer and Peter with Cambridge Assessment where he was an advisory consultant on secondment from his specialist work with the British Council). Such a unique relationship in assessment and indepth understanding of the Hong Kong context is reflected in the coherence of this programme of research.

Validating Technological Innovation: The Introduction and Implementation of Onscreen Marking in Hong Kong not only brings the most recent work on OSM situated in a unique context of education but also makes many new and significant contributions to the field, which I believe have the potential to help test developers and test users all over the world.

Faculty of Education, Queen's University

Liying Cheng

Kingston, ON, Canada

\section{Reference}

Bachman, L.F. (2000). Modern language testing at the turn of the century: Assuring that what we count counts. Language Testing, 17(1), 1-42. 



\section{Acknowledgements}

We are grateful to a number of people whose efforts, scholarship and expertise made this book possible.

Christina Lee of the Hong Kong Examinations and Assessment Authority (HKEAA) provided her expertise and experience to create and write Chaps. 2 and 3. Chapter 2 'The Role of the Hong Kong Examinations and Assessment Authority' introduces the reader to the background to the development and implementation of OSM in Hong Kong and describes the processes that the innovation went through. In Chap. 3, 'Onscreen Marking System', Christina, through the use of screen-grabs demonstrates for the reader what the markers physically see on the screen as they process and mark scripts and record their marks.

We are also grateful to Alison Sau-chu Yeung (Chap. 7) and Zi Yan (Chaps. 10, 12, and 13) for their contributions to papers that they co-wrote with the authors and for their consent to publish their papers.

As we acknowledge in the footnote to Chap. 4, we are grateful to Julie Mackay of Cambridge Assessment and Sarah Phillips of RM Results for their efforts in securing us details of Cambridge examinations marked by means of OSM. In terms of the provision of relevant research information, we are grateful to Ardeshir Geranpayeh of Cambridge Assessment for his links to relevant Cambridge-based research.

Finally, we would like to thank Professor Liying Cheng of Queens University, Kingston, Ontario, for writing a Foreword. She is an extremely busy academic, working in the field of examinations and assessment, and we are grateful to her for her efforts at a particularly busy time in her work cycle. 



\section{Contents}

1 The Hong Kong Education and Assessment Systems . . . . . . . . 1

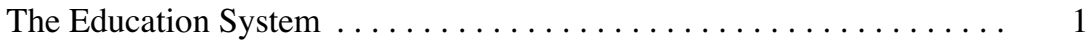

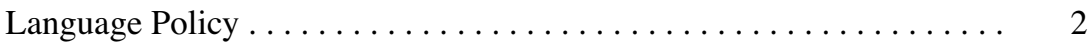

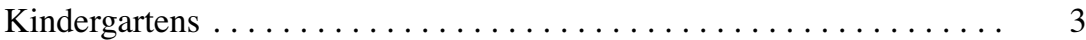

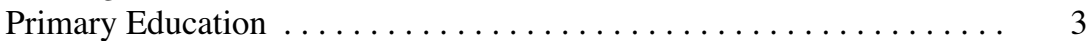

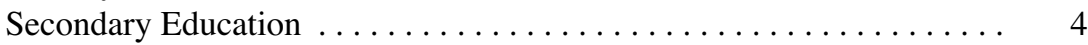

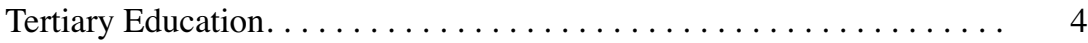

The Hong Kong Diploma of Secondary Education (HKDSE) . . . . . . 5

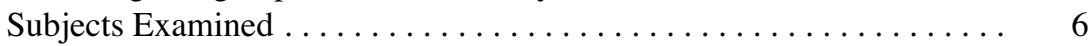

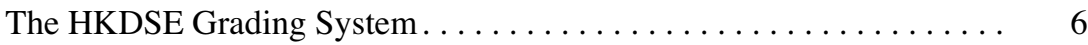

Summary................................ 7

2 The Role of the Hong Kong Examinations

and Assessment Authority $\ldots \ldots \ldots \ldots \ldots \ldots \ldots \ldots \ldots$

The Hong Kong Examinations and Assessment Authority. . . . . . . . . . 9

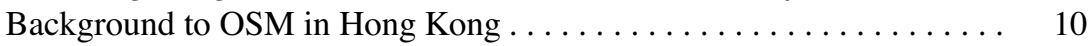

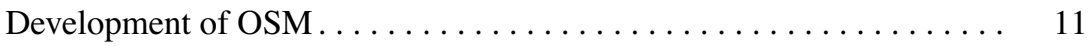

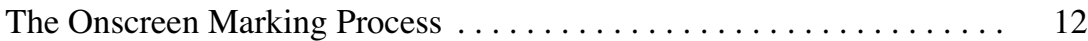

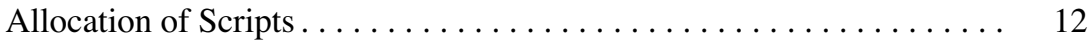

Marking and Check-Marking Procedures............... 12

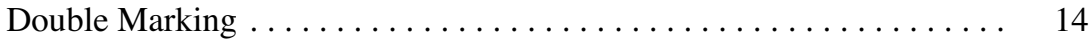

Benefits and Drawbacks of OSM . . . . . . . . . . . . . . 14

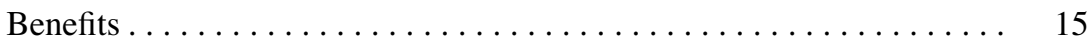

Drawbacks. . . . . . . . . . . . . . . . . . . . . . . 15

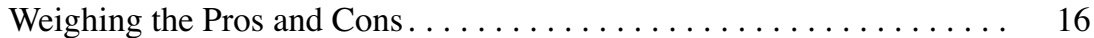

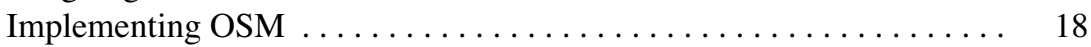

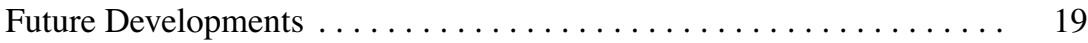

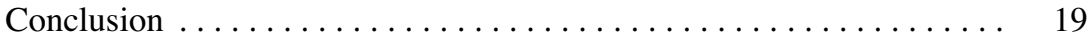

Appendix 1: Selected HKEAA Milestones. . . . . . . . . . . . 20 
3 Onscreen Marking System ........................ 23

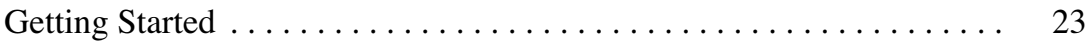

Logging into the OSM System ...................... 24

Viewing Reference Scripts ....................... 25

Viewing Marking Summary ....................... 27

Marking. .................................... 28

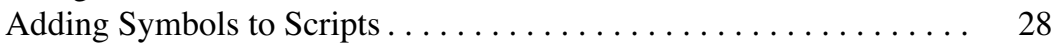

Inserting Comments into Scripts.................... 30

Awarding Marks to Scripts ....................... 31

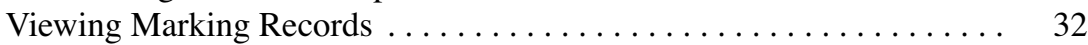

Viewing Marking Statistics ....................... 34

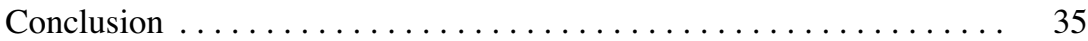

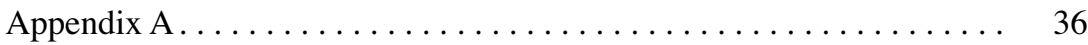

The Graduating Students' Language Proficiency

Assessment (GSLPA) OnScreen Rating System (ORS) . . . . . . . . 36

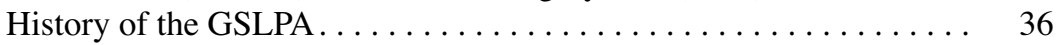

The GSPLA. ............................. 37

4 Review of Research into Onscreen Marking and a Description of the Methodological Approaches and Analytical Tools Used in the OSM Validation Studies ....... 43

Part 1: A Review of the Research Literature on OSM ........... 43

Part 2: Theoretical Underpinning, Methodological

Approaches and Analytical Tools ...................... 48

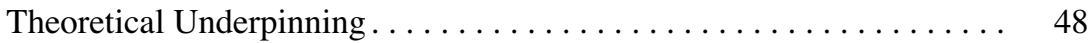

Methodological Approaches ........................... 49

Quantitative Survey Approach . . . . . . . . . . . . . . . . . 49

Qualitative Approach Including a Grounded Theory Approach..... . 49

Methodological Tools ............................. $\quad 50$

Parametric Tests ................................. 50

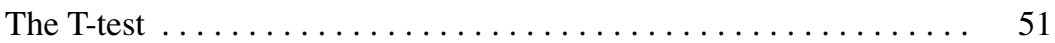

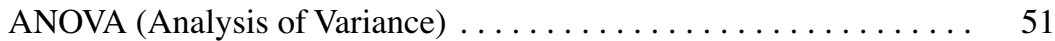

The Pearson Product-Moment Correlation............... 51

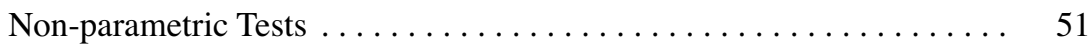

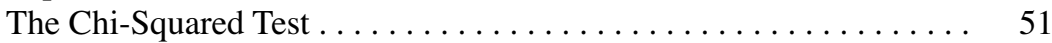

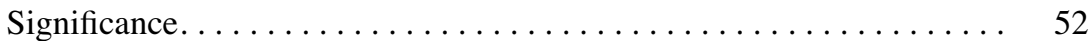

The Rasch Model .............................. 52

Many-Facet Rasch Measurement (MFRM) and Data Analysis ...... 53

MFRM Analysis: Results and Discussion. . . . . . . . . . . . . . . 54

Qualitative Data Analysis . . . . . . . . . . . . . . . . . . 55

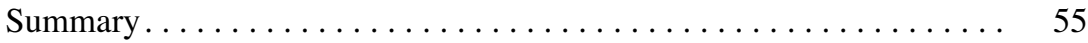


5 The First Validation Study: Comparing OSM and PBM in English Language HKCEE (Year 11) Essays Using Classical Statistics and Rasch Measurement. . . . . . . 57

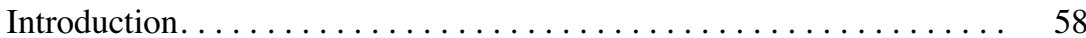

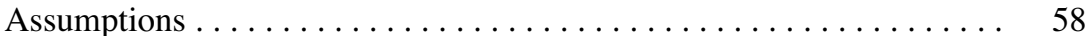

The Research. . . . . . . . . . . . . . . . . . . . . . . . . . . . . 59

Data................................. 59

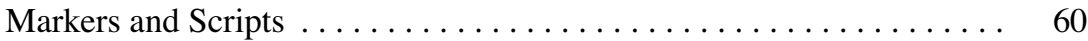

Background Details of the Markers ................. 61

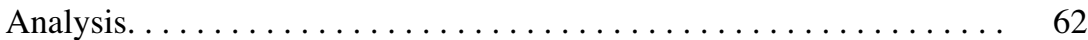

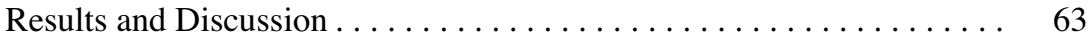

Computer Proficiency . . . . . . . . . . . . . . . . . . . . . . . 63

OSM and PBM Marking Issues $\ldots \ldots \ldots \ldots \ldots \ldots \ldots \ldots \ldots \ldots$

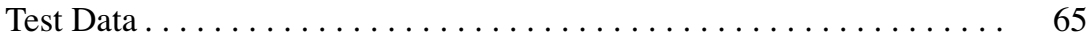

Writing Paper Prompts . . . . . . . . . . . . . . . . . 66

Inter-marker Reliability Correlations . . . . . . . . . . . . . 67

Marker Experience Status as a Grouping Variable . . . . . . . . . . . . 67

Discrepancies Between the Two Forms of Rating . . . . . . . . . . 68

Many-Faceted Rasch Measurement (MFRM)

and Data Analysis . . . . . . . . . . . . . . . . . . . . . . 69

MFRM Analysis: Results and Discussion. . . . . . . . . . . . . . 69

Conclusion . . . . . . . . . . . . . . . . . . . . . . . . . 73

Notes . . . . . . . . . . . . . . . . . . . . . . . . . 75

Appendix 1: 2007 HKCEE English Language

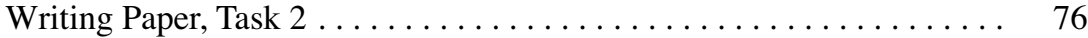

Appendix 2: Onscreen Marking Questionnaire - 2007

HKCEE English Language Writing Paper . . . . . . . . . . . 76

6 The Second Validation Study: A Qualitative Study

of the Response of Markers Towards Onscreen

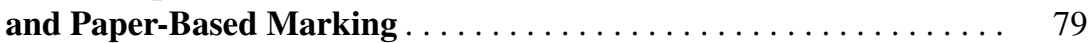

Introduction. . . . . . . . . . . . . . . . . . . . . . . . . . . . . . . 79

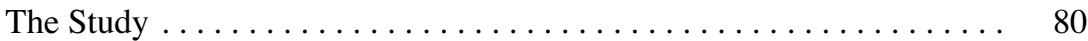

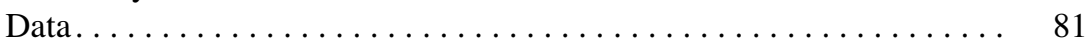

Semi-structured Interviews: Standardisation of Interviewers . . . . . . . . 81

Results and Discussion ..................... 82

IT Proficiency and the Ability to Cope with the Technical

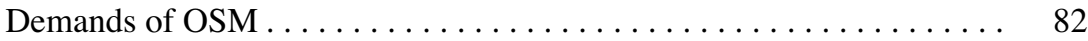

Reading on Screen ........................ 83

Views on the Reliability and Efficiency of Marking by OSM . . . . . . 84

Re-reading of Scripts $\ldots \ldots \ldots \ldots \ldots \ldots \ldots \ldots \ldots \ldots \ldots \ldots \ldots$

Training and Standardisation $\ldots \ldots \ldots \ldots \ldots \ldots \ldots \ldots \ldots \ldots$

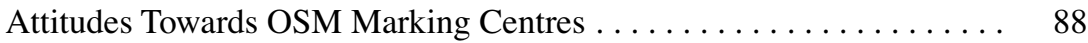

Views on Working from Home . . . . . . . . . . . . . . . . . 89

Conclusion ............................. 90 
Appendix 1: Interviewees' Profiles . . . . . . . . . . . . . . 91

Appendix 2: Checklist of Guideline Questions

for the Semi-structured Interviews . . . . . . . . . . . . . . . . . . . . . . 92

7 The Third Validation Study: Markers' Perceptions

of the Onscreen Marking of Liberal Studies

in the Hong Kong Public Examination System . . . . . . . . . . . . 93

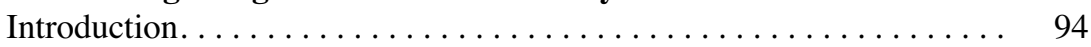

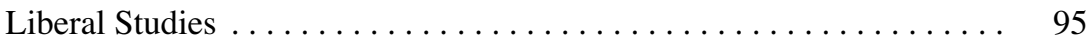

Research Questions. .......................... 96

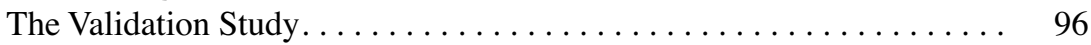

Results.............................. 97

Markers, Test Takers and Modules . . . . . . . . . . . . . . . . . . 97

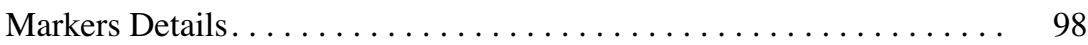

Pre-marking Questionnaire . . . . . . . . . . . . . . . . . 99

Post-Marking Questionnaire . . . . . . . . . . . . . . . . . . . . 101

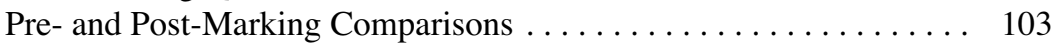

Conclusion .............................. 104

Appendix 1: 2009 ASL Liberal Studies: Onscreen

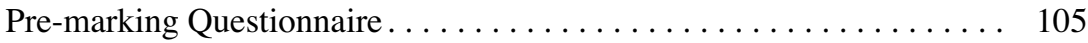

Appendix 2: 2009 ASL Liberal Studies: Onscreen

Post-Marking Questionnaire...................... 107

8 The Fourth Validation Study: A Qualitative Examination

of the Attitudes of Liberal Studies (LS) Markers

Towards Onscreen Marking in Hong Kong . . . . . . . . . . . . . . . 109

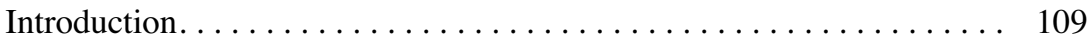

The Qualitative Study . . . . . . . . . . . . . . . . . . 110

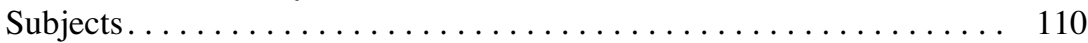

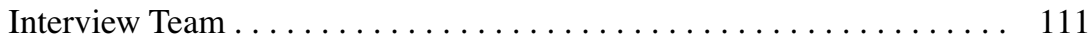

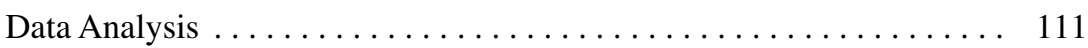

Results.............................. 112

Computer Hardware and Software . . . . . . . . . . . . . . . . 112

Marking Centres: The Environment, Location and Booking . . . . . . 114

Marker Training, Support and Standardisation. . . . . . . . . . . 115

Marking-Related Issues . . . . . . . . . . . . . . . . . . . . 116

Discussion............................ 118

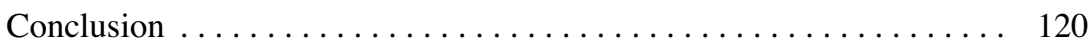

Notes . . . . . . . . . . . . . . . . . . . . . . . . . . 120

Appendix 1: Participant Profiles . . . . . . . . . . . . . . 121

9 The Fifth Validation Study: Examining Negative

Attitudes Towards OSM in Hong Kong . . . . . . . . . . . . . . . . . . 123

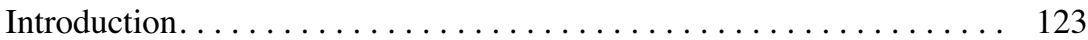

Impetus for the Current Study. . . . . . . . . . . . . . . . . . 124

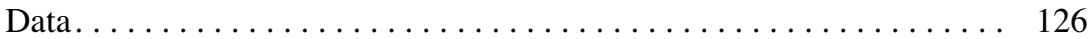

Results and Discussion . . . . . . . . . . . . . . 126 
Scores Awarded to Test Takers . . . . . . . . . . . . . . . . . . . . 127

Marker Attitude as a Grouping Variable . . . . . . . . . . . . . . . . . 127

Correlations Between 2007 HKCEE English Language Papers. . . . . . 128

Marker Erraticness . . . . . . . . . . . . . . . . . . . . . . . . . 129

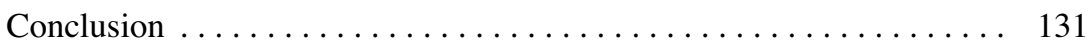

Note. . . . . . . . . . . . . . . . . . . . . . . . . . . . . . 132

10 The Sixth Validation Study: Assessing the Ease

of Use in the Environment and Markers' Acceptance

of Onscreen Marking in Hong Kong in Three

Subject Areas: A Rasch Measurement Perspective . . . . . . . . . . 133

Introduction. . . . . . . . . . . . . . . . . . . . . . . 134

The Rasch Model . . . . . . . . . . . . . . . . . . . . . . . . . . . . . . . . 136

Background to the Study. . . . . . . . . . . . . . . . . 137

The Study . . . . . . . . . . . . . . . . . . . . . . . . . . . 138

Research Questions. . . . . . . . . . . . . . . . . . . . . . . . 139

Data Analysis . . . . . . . . . . . . . . . . . . . . . . . . . . . 140

Language, Question Type, and Subject of Scripts . . . . . . . . . . . 141

Results and Discussion . . . . . . . . . . . . . . . . . 141

Ease of Use in the OSM Environment Scale . . . . . . . . . . . . . . 141

Acceptance of OSM Scale . . . . . . . . . . . . . . . . . . 144

Person Measures on the Ease of Use in the OSM Environment

and Acceptance of OSM Scales . . . . . . . . . . . . . . . . . . . . 145

Comparisons of Mean Person Measures. . . . . . . . . . . . . . . . 148

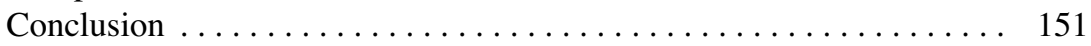

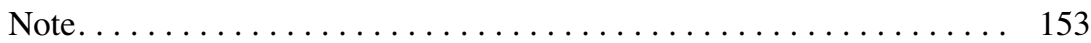

Appendix 1: 2012 HKALE/HKDSE: Onscreen

Post-Marking Questionnaire ... . . . . . . . . . . . . . . . 153

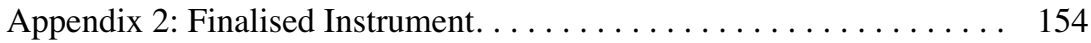

11 The Seventh Validation Study: The Increasing

Acceptance of Onscreen Marking: The 'iPad Effect' . . . . . . . . . . 157

Introduction. . . . . . . . . . . . . . . . . . . . . . . . . . . 158

Tablet Computers: Background and Their Uptake

in the Field of Education. . . . . . . . . . . . . . . . . . . 159

The Study . . . . . . . . . . . . . . . . . . . . . . . . . . . 160

Research Questions........................... 162

Results and Discussion ..................... 162

Markers, Test Takers and Modules . . . . . . . . . . . . . . . . 163

Owning a Tablet ... . . . . . . . . . . . . . . . . . . . . . . . . 164

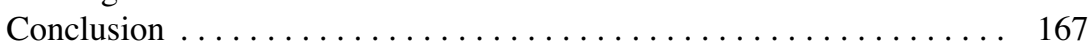

Note. . . . . . . . . . . . . . . . . . . . . . . . . . 168

2012 HKAL/HKDSE: Onscreen Pre-marking

Questionnaire: Marking Assistants . . . . . . . . . . . . . . . . . . . 169

2012 HKAL/HKDSE: Onscreen Post-Marking Questionnaire:

Marking Assistants . . . . . . . . . . . . . . . . . . . . . . . . . . . . 169 
12 The Eighth Validation Study: The Effects of Key

Demographic Variables on Markers' Perceived Ease of Use and Acceptance of Onscreen Marking . . . . . . . . . . . 171

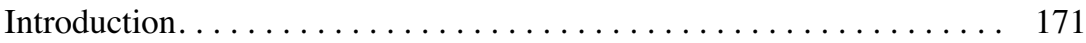

The Study . . . . . . . . . . . . . . . . . . . . . . . . . . . 174

Dataset. ..................................... 174

Research Questions............................ 175

Methodology ................................ 175

Instrument................................... 175

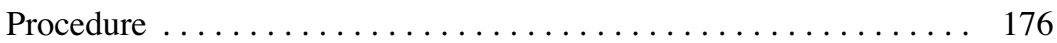

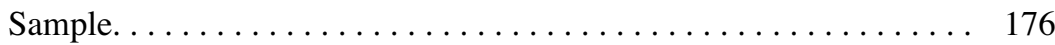

Results and Discussion ......................... 177

The Psychometric Properties of the Two Scales

for Use with the Sample in the Current Study . . . . . . . . . . . . 177

Markers' Responses to the Ease of Use in the OSM

Environment and Acceptance of OSM Scales .............. 178

Effects of Language of Marking, Gender, Age on Markers

Reactions to OSM............................... 179

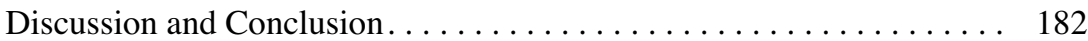

Notes .................................... 185

Appendix 1: Onscreen Marking Questionnaire ............ 185

Appendix 2: Item Fit Statistics, Item Measures

with Standard Errors (in parentheses), and Category Thresholds. . . . . 186

Appendix 3: Marker-Item Maps ...................... 186

\section{The Final Validation Study: A Comparative Picture}

of the Ease of Use and Acceptance of Onscreen

Marking by Markers Across Subject Areas. . . . . . . . . . . . . . . 189

Introduction. . . . . . . . . . . . . . . . . . . . . . . . . . . . . 190

Research Questions. . . . . . . . . . . . . . . . . . . . . . . . . . 191

Methodology.................................. 191

Responses to the Likert-Type Questionnaire . . . . . . . . . . . . . 192

Open-Ended Comments . . . . . . . . . . . . . . . . . . . . . . . . . . . . . . . . . . . 194

Interviews with Key HKEAA Personnel . . . . . . . . . . . . . . . . . 194

Results and Discussion ............................ 195

The Psychometric Properties of the Two Scales. . . . . . . . . . . . 195

Cross-Subject Area Comparison. . . . . . . . . . . . . . . . . . . . . . . . . 195

Marker Measures on the Two Scales . . . . . . . . . . . . . . . . 196

Analysis of Written Comments. . . . . . . . . . . . . . . . . . . . . . 199

Interview with HKEAA Personnel . . . . . . . . . . . . . . . . 201

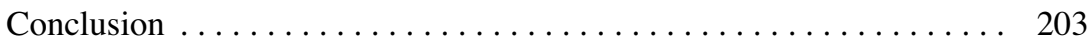

Notes................................. 205

Appendix 1: HKDSE, HKALE Subject Areas

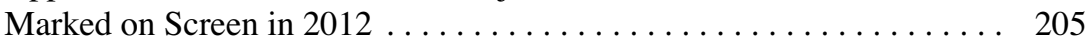




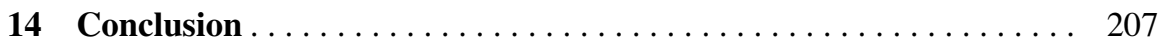

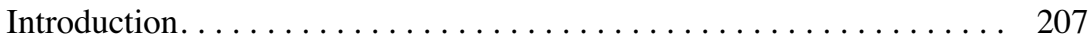

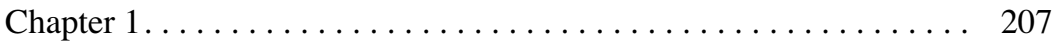

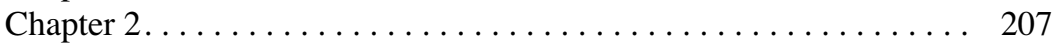

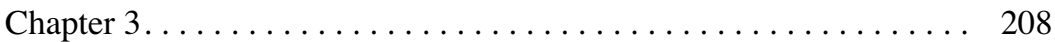

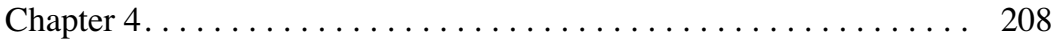

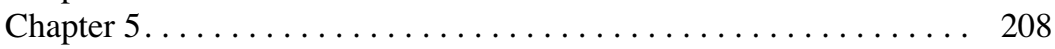

Chapter 6.............................. 208

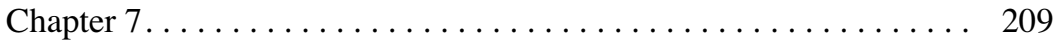

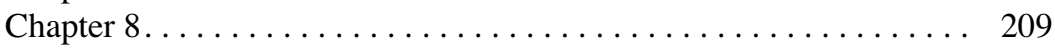

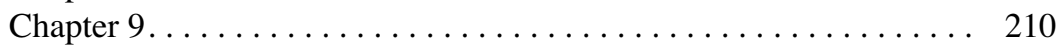

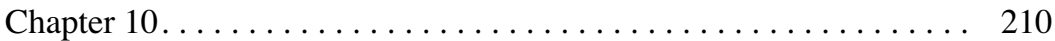

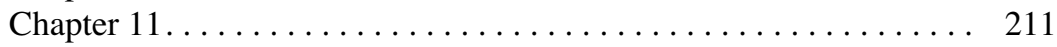

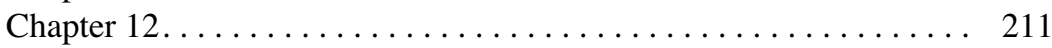

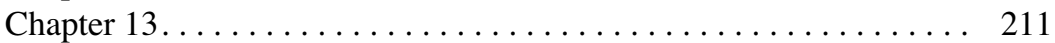

Weaknesses Identified with the Implementation

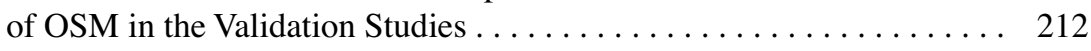

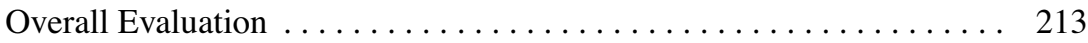

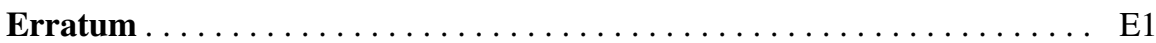

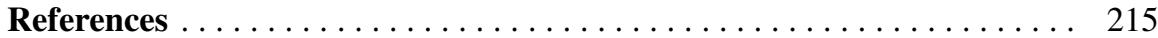

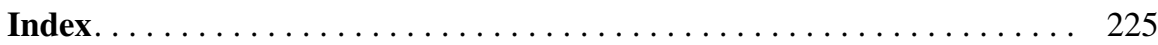





\section{Contributors}

Liying Cheng is Professor and Head of the Assessment and Evaluation Group at the Faculty of Education, Queen's University at Kingston Ontario, Canada. Her primary research interests are the impact of large-scale testing on instruction and the relationships between assessment and instruction.

David Coniam is Chair Professor of Curriculum and Assessment and head of the Department of Curriculum and Instruction in the Faculty of Education and Human Development at The Education University of Hong Kong, where he is a teacher educator, working with teachers in Hong Kong primary and secondary schools. His main publication and research interests are in language assessment, language teaching methodology and computer-assisted language learning.

Peter Falvey is a teacher educator. A former head of department in the Faculty of Education, the University of Hong Kong; he is currently affiliated in an honorary professorial role with The Education University of Hong Kong. His main publication and research interests are in language assessment, first and second language writing methodology and text linguistics.

Christina Lee is Director of Public Examinations at the HKEAA. She leads the development and administration of the Hong Kong Diploma of Secondary Education (HKDSE) and is responsible for the implementation, review and enhancement of public examination policies and procedures. She led the onscreen marking (OSM) project and played an important role in the introduction of OSM in 2007 and its subsequent across-the-board implementation in the HKDSE.

Alison Sau-chu Yeung is Associate Professor of practice in the Department of Curriculum and Instruction in the Faculty of Education at the Chinese University of Hong Kong, where she offers courses on the subject and curriculum teaching of Chinese history, world history, liberal studies and general studies. Her research interests include history education, subject culture, teacher education, teachers' narratives and women's history. 
Zi Yan is Assistant Professor and associate head of the Department of Curriculum and Instruction in the Faculty of Education and Human Development at The Education University of Hong Kong. His main publication and research interests are in educational and psychological assessment and Rasch measurement. 


\section{Common Abbreviations Used in the Book}

$\begin{array}{ll}\text { BAFS } & \text { Business, accounting and financial studies } \\ \text { CMI } & \text { Chinese medium of instruction } \\ \text { CTT } & \text { Classical test theory } \\ \text { DIF } & \text { Differential item functioning } \\ \text { EMI } & \text { English medium of instruction } \\ \text { 'gaokao' } & \text { National College entrance examination } \\ \text { GSLPA } & \text { Graduating students' language proficiency assessment } \\ \text { HKALE } & \text { Hong Kong advanced level examinations } \\ \text { HKCEE } & \text { Hong Kong certificate of education examination } \\ \text { HKDSE } & \text { Hong Kong diploma of secondary education } \\ \text { HKEAA } & \text { Hong Kong examination and assessment authority } \\ \text { HKIEd } & \text { Hong Kong Institute of Education } \\ \text { HKSAR } & \text { Hong Kong Special Administrative Region } \\ \text { ICT } & \text { Information and communication technology } \\ \text { IELTS } & \text { International English language testing system } \\ \text { LPAT } & \text { Language proficiency assessment for teachers } \\ \text { LS } & \text { Liberal studies } \\ \text { MFRM } & \text { Many-Facet Rasch measurement } \\ \text { OSM } & \text { Onscreen marking } \\ \text { PBM } & \text { Paper-based marking } \\ \text { TSA } & \text { Territory-wide system assessment } \\ \text { UEC } & \text { HKALE use of English examination } \\ & \end{array}$





\title{
Introduction
}

\begin{abstract}
This introductory chapter introduces the reader to our purpose in writing this book, viz. to provide a coherent account of the design and implementation of onscreen marking of examination scripts in public examinations in Hong Kong by linking a number of validation studies between 2009 and 2012. We explain why we chose to take the nine validations studies on OSM which had been previously published and bind them together into a coherent whole by surrounding them with an explanatory background to Hong Kong's educational and assessment history and by setting out relevant research in this area from around the world. A short description of each chapter is then provided.
\end{abstract}

\section{Overview of Book Structure}

The purpose of this book is to provide those involved in public examinations and other scholars interested in assessment and evaluation with a coherent account of a series of validation studies linked to the implementation of onscreen marking (OSM) in Hong Kong. Onscreen marking (the marking of examination scripts on screen instead of on paper) is being used more and more often as technology develops. This series of validation studies began, in 2009, with the publication of Coniam (2009a), the first description of what became, by 2014/2015, a series of validation studies of OSM in the school public examinations system in Hong Kong, operated by the Hong Kong Examinations and Assessment Authority (HKEAA). In all, a total of nine validation studies were carried out and published by David Coniam, sometimes with co-writers (Alison Sau-chu Yeung, Zi Yan and Peter Falvey, the coauthor of this book). The co-authors and the publications in which the articles originally appeared are provided at the end of each abstract in the descriptions of the relevant chapters below.

It quickly became apparent to the authors that a number of assessment scholars were unable to access all the papers easily as a coherent whole. After discussion, the authors agreed to consolidate the validation studies in the production of a book 
consisting of 14 chapters. The four opening chapters would be completely new. Chapter 1, entitled 'The Hong Kong Education and Assessment Systems', helps to orientate readers to the history of Hong Kong's education system and its curricula in addition to its changing assessment curricula, particularly the wide-ranging changes that occurred after Hong Kong was handed back to China in 1997. Chapter 2, written by Christina Lee of the HKEAA provides an account of the role of the HKEAA in the process of inaugurating, developing and implementing OSM. Lee makes clear our relationship with the HKEAA, and that the research studies we conducted were conducted independently and not associated with, sponsored by or funded by the HKEAA. Chapter 3 uses screen grabs to pictorially help the reader understand the processes that markers go through when undergoing training, qualifying and live marking on OSM. Chapter 4, entitled 'Review of Research into Onscreen Marking and a Description of the Methodological Approaches and Analytical Tools Used in the Validation Studies, describes two things: the research literature on OSM and the methodological approaches used in the validation studies together with a description of the analytical tools used in the data analysis. The chapter also describes how the book is unique.

After the four opening chapters, the validation studies are presented in Chaps. 5, $6,7,8,9,10,11,12$, and 13 which update, refresh and where necessary revise and re-write the original nine journal articles in such a way as to reveal the processes that occurred during the initiation and completion of the studies and to make that process coherent to the reader. The revising of the chapters makes clear the links between the chapters and the events/reactions from stakeholders that influenced the direction of subsequent studies and how they were reported in subsequent chapters, e.g. enlarging studies from one subject area to more than one subject area to, eventually, coverage of all subjects in Chap. 13 and, for example, reacting to stakeholders' views in the study described in Chap. 9. Chapter 14 summarises what has gone before and concludes the book.

\section{Chapters 5, 6, 7, 8, 9, 10, 11, 12, and 13: The Validation Studies}

This section presents an overview of the contents of Chaps. 5, 6, 7, 8, 9, 10, 11, 12, and 13 .

\section{Chapter 5}

Chapter 5 describes the first validation study. It is entitled 'The First Study: Comparing OSM and PBM (Paper-Based Marking) in English Language HKCEE (Year 11) Essays Using Classical Statistics and Rasch Measurement'. There were four hypotheses: two related to marker attitude and two to statistics. The first was 
that markers would be sufficiently competent technologically to function effectively within the new OSM medium. The second was that markers would not be negative about the OSM medium, showing no preference for either marking medium. These hypotheses investigated the crucial issues of technological competence and marker attitudes towards OSM, without which the implementation of OSM would be invalid and marker attitudes could affect the successful implementation of the innovation. Overwhelmingly negative attitudes towards OSM could have affected the validity of the innovation.

The third and most essential hypothesis was that inter-marker reliability between the two marking mediums would be comparable to inter-marker reliability achieved between markers marking on paper. The fourth, equally essential hypothesis was that the marking medium would not affect scores awarded to test takers, with test takers obtaining the same score whether their scripts were marked on screen or on paper. These two hypotheses were critical in that stakeholder confidence would be badly shaken if inter-marker reliability was not comparable and if different scores were to be awarded in paper-based marking and onscreen marking.

This study originally appeared as: Coniam, D. (2009). A comparison of onscreen and paper-based marking in the Hong Kong public examination system. Educational Research and Evaluation. 15(3), 243-263.

\section{Chapter 6}

Chapter 6 is entitled 'A Qualitative Study of the Response of Markers Towards Onscreen and Paper-Based Marking'. It uses the same markers as the study in Chap. 5. It investigates the in-depth responses not only of those who had experience of OSM but of those who formerly had marked only on paper. It discusses marker attitudes towards various issues. The first topic investigates IT proficiency and the markers' ability to cope with the technical demands of OSM. This is followed by five other issues that arose during the semi-structured interviews, some of which had not been revealed in the quantitative survey described in Chap. 5, viz. reading on screen, views on the reliability and efficiency of marking by OSM, training and standardisation, attitudes towards OSM marking centres and views on working from home.

This study originally appeared as: Falvey, P. and Coniam, D. (2010). A qualitative study of the response of markers towards onscreen and paper-based marking. Melbourne Papers in Language Testing, 15(1), 1-26.

\section{Chapter 7}

Chapter 7 is entitled 'Markers' Perceptions of the Onscreen Marking of Liberal Studies in the Hong Kong Public Examination System'. The hypotheses in this quantitatively based study relate to the attitudes of markers of liberal studies (LS) to 
OSM. It moves the series on from a study of English language markers to liberal studies markers. The first hypothesis is that markers will judge themselves to be sufficiently competent technologically to function effectively within the new OSM medium. The second hypothesis is that markers will not be negative about the OSM medium and will show no preference for either marking medium. The results of the study indicated that OSM was becoming accepted into marker psyche of what marking involves.

This study first appeared as: Coniam, D. and Yeung, A. (2010). Markers' perceptions regarding the onscreen marking of Liberal Studies in the Hong Kong public examination system. Asia Pacific Journal of Education, 30(3), 249-271.

\section{Chapter 8}

The study reported in Chap. 8 is entitled A Qualitative Examination of the Attitudes of Liberal Studies Markers Towards Onscreen Marking in Hong Kong'. Similar to the qualitative study described in Chapter 6, this study is also a qualitative investigation that complements the quantitative study of liberal studies outlined in Chap. 7. It describes markers' attitudes and insights through the use of 'thick' description (Geertz 1973).

Chapter 8 reports on the detailed responses of LS markers in semi-structured interviews. The study investigates markers' perceptions of the benefits and drawbacks of the new OSM system from four perspectives: The four major areas were:

1. Computer hardware and software

2. Marking centres: environment, location and booking

3. Marker training, support and standardisation

4. Marking-related issues

A number of new findings emerged from the qualitative data. One new finding involved the use of the Internet to check the accuracy of student content and/or plagiarism - potentially an interesting issue for the HKEAA. The most frequent concluding comment was the need for more marking centres. It would appear from the results that, despite its present shortcomings, the potential of OSM as a viable system was being recognised and generally accepted.

This study on which this chapter is based first appeared as: Coniam, D. (2011). A qualitative examination of the attitudes of Liberal Studies markers towards onscreen marking. British Journal of Educational Technology, 42(6), 1042-1054.

\section{Chapter 9}

Chapter 9 is entitled 'Examining Negative Attitudes Towards OSM in Hong Kong'. The study extends the second research question from the study described in Chap. 5. In that study, markers completed a questionnaire detailing their attitudes towards 
the onscreen and paper-based marking processes. In terms of attitudes between the two sets of markers - old and new - there were clear differences. In terms of general preference for onscreen versus paper-based marking, new markers rated OSM more positively than experienced markers. Also, in terms of a preference for marking at home or at a centre, new markers rated centre marking much more positively than did experienced markers; new markers also felt that having to travel to a special marking centre was less of an inconvenience than that endured by experienced markers. The details of the study described in Chap. 5 were presented to the HKEAA Research and Development Committee in early March 2009. The Committee accepted the report, but raised concerns about negative Hong Kong teacher marker attitudes towards the general adoption of OSM. The Committee had concerns about the attitude of these disaffected markers and wondered whether those attitudes might be reflected in the scores awarded to test takers. These concerns provided the impetus for the study described in this chapter. The stated hypothesis in Chap. 5 that 'markers will not be negative about the OSM medium, showing no preference for either marking medium', could be neither proved nor disproved, although the tendency was for a more negative than positive orientation.

As stated above, the study in Chap. 9 extended the second research question from the original study and investigated whether markers' attitudes affected the OSM mark awarded. Specifically, it pursued two hypotheses:

1. That markers holding a negative attitude towards OSM would rate test takers more harshly than would markers who have a positive attitude

2. That markers holding a negative attitude towards OSM would be more erratic than more positively oriented markers

Clearly, if both hypotheses were accepted, there would be a validity issue with OSM when faced with negative markers whose marks were affected by their attitude to OSM. This chapter describes what those reactions were.

This study originally appeared as: Coniam, D. (2010). Examining negative attitudes towards onscreen marking in Hong Kong. Education Journal, 37(1-2), 71-87.

\section{Chapter 10}

The study described in Chap. 10 is entitled 'Assessing the Ease of Use in the Environment and Markers' Acceptance of Onscreen Marking in Hong Kong in Three Subject Areas: A Rasch Measurement Perspective'. The study expanded the data base of English language and liberal studies markers by including the entire set of scripts from three subject areas: liberal studies, English language and Chinese language. LS scripts comprised extended essays, while English language and Chinese language scripts comprised the sections requiring short-answer questions. In addition, there was a language factor in the study, with candidate scripts written in English or Chinese. 
With this heterogeneous background - and with the overriding objective of calibrating a robust instrument for use in a future large-scale study (see Chap. 13) there were two linked sets of hypotheses: one set for ease of use in the OSM environment and another for acceptance of OSM. These were as follows:

1. Concerning ease of use in the OSM environment, the item difficulties will not differ for markers with regards to:

- The language (English or Chinese) that scripts are marked in

- The type of question (extended essays or short-answer questions)

- The subject (English or Chinese or LS)

2. Concerning acceptance of OSM, the item difficulties will not differ for markers with regards to:

- The language (English or Chinese) that scripts are marked in

- The type of question (extended essays or short-answer questions)

- The subject (English or Chinese or LS)

The statistical approach in this study involved the use of Rasch measurement. Such an approach not only enabled the research questions above to be explored, it also permitted a robust scale with sound psychometric properties to be established from the items in the questionnaire.

This study originally appeared as: Yan, Z. and Coniam, D (2013). Assessing the ease of use in the environment and markers' acceptance of on screen marking: a Rasch measurement perspective. Educational Research and Evaluation, 19(5), 461-483.

\section{Chapter 11}

Chapter 11 is entitled 'The Increasing Acceptance of Onscreen Marking: The "Tablet Computer" Effect'. In this study, the marker sample was different from previous studies in that the subjects were younger markers who mainly had not previously marked for the HKEAA. In addition, these markers were marking for an extended period $-6 \mathrm{~h}$ or more in a single day. The study pursued the hypothesis that younger markers would, as a starting point, be more predisposed to marking on screen than would older markers. The study also hypothesised that, with the massive take-up of tablets, the younger generation of markers would be even more predisposed towards OSM, with ownership of a tablet resulting in a more positive attitude towards OSM.

There were, consequently, three hypotheses in the study which relate to marker attitude:

1. Markers of the short answers in the 2012 Hong Kong Advanced Level Use of English Examination would be more positive towards the OSM medium in their post-marking questionnaires than were HKALE Liberal Studies markers in the 2010 study. 
2. Markers would be more positive towards the OSM medium in their post-marking questionnaire than in their pre-marking questionnaire.

3. Markers who personally owned a tablet would have a more positive attitude towards the OSM marking medium in both their pre- and post-marking questionnaires than markers who did not own a tablet.

This study originally appeared as: Coniam, D. (2013). The increasing acceptance of onscreen marking - the 'tablet computer' effect. Journal of Educational Technology \& Society, 16(3), 119-129).

\section{Chapter 12}

Chapter 12 is entitled 'The Effects of Key Demographic Variables on Markers' Perceived Ease of Use and Acceptance of Onscreen Marking'. The major purpose of this study was to investigate the extent to which the different languages used to mark, gender and age affected markers' perceived ease of use in the OSM environment and acceptance of OSM. The research questions were formulated as follows:

1. What are the effects of the language of marking (English, Chinese or both), gender, and age on markers' perceived ease of use in the OSM environment?

2. What are the effects of the language of marking (English, Chinese or both), gender, and age on markers' perceived acceptance of OSM?

This study originally appeared, in a slightly different version, as: Yan, Z., and Coniam, D. (2014). The effects of key demographic variables on markers' perceived ease of use and acceptance of onscreen marking. Assessment in Education: Principles, Policy \& Practice, 21(4), 464-480.

\section{Chapter 13}

Chapter 13 is entitled 'A Comparative Picture of the Ease of Use and Acceptance of Onscreen Marking by Markers Across Subject Areas'. The major focus involved comparing marker reactions from the wider perspective of all the subject areas marked by OSM for the first time in 2012. The research questions were framed as:

1. What are the effects of subject area on markers' perceived ease of use in the OSM environment?

2. What are the effects of subject area on markers' perceived acceptance of OSM?

Chapter 13 describes the final study in the series of validation studies described in the book. It recounts how all subject areas were marked by OSM in the 2012 administration of Hong Kong's public school examinations. It was thus a fitting opportunity to see whether, overall, OSM worked in terms of validity for future use. 
This study originally appeared as: Coniam, David and Yan, Zi. (2015). A comparative picture of the ease of use and acceptance of onscreen marking by markers across subject areas. British Journal of Educational Technology, doi: 10.1111/bjet.12294.

\section{Chapter 14}

The book ends with the concluding chapter, Chap. 14, that summarises the studies that were described throughout the book and discusses the major findings in the studies, any weaknesses or problems that were detected which would need to be faced by HKEAA, and the overall findings and outcomes of the studies.

An important element within the book is the ways in which feedback from stakeholders was used to initiate further studies, as in Chap. 8, for example, where concerns of stakeholders that dissatisfied markers might not mark as accurately or reliably as non-dissatisfied markers were addressed in a qualitative, in-depth research study.

It should be noted that apart from wishing to provide an accessible, coherent account of the nine validation studies, the authors also wanted to provide stakeholders in various jurisdictions worldwide with potential templates for validating their own OSM processes and implementations by replicating the Hong Kong validation studies.

Note: It should be noted that the term 'marker' is used throughout the book although the term 'rater' was used in some of the papers which have been revised and re-written in Chaps. 5, 6, 7, 8, 9, 10, 11, 12, and 13. The reason for this is that the term 'marker' has sometimes been used to refer to those who award marks for short-answer or multiple-choice answers and 'rater' has sometimes been used to refer to those who mark performance examinations, e.g. those that involve writing and speaking. We have chosen not to use that distinction. Thus, we have chosen to use the one term 'marker' only.

Tai Po, Hong Kong

David Coniam Peter Falvey

\section{Reference}

Coniam, D. (2009a). A comparison of onscreen and paper-based marking in the Hong Kong public examination system. Educational Research and Evaluation, 15(3), 243-263.

Geertz, C. (1973). Thick description: Toward an interpretative theory of culture. In The interpretation of cultures: Selected essays (pp. 3-30). New York: Basic Books.

The original version of the Front Matter was revised. Editors and Contributors affiliations was updated. An erratum can be found at http://dx.doi.org/10.1007/978-981-10-0434-6_15 